\title{
Diagnostic Algorithm for Conjunctival Melanocytic Lesions
}

\author{
MAGDALENA DĘBICKA-KUMELA ${ }^{1,2}$, BOZENA ROMANOWSKA-DIXON ${ }^{1,2}$ IZABELLA KARSKA-BASTA $^{1,2}$, \\ JOANNA KOWAL ${ }^{1,2}$, WERONIKA POCIEJ-MARCIAK ${ }^{1,2}$ and ANNA MARKIEWICZ ${ }^{1,2}$ \\ ${ }^{1}$ Department of Ophthalmology and Ocular Oncology, Faculty of Medicine, \\ Jagiellonian University Medical College, Krakow, Poland; \\ ${ }^{2}$ Department of Ophthalmology and Ocular Oncology, University Hospital, Krakow, Poland
}

\begin{abstract}
Background/Aim: This study aimed to develop an algorithm allowing the differentiation between conjunctival melanoma and other melanocytic infiltrations of the conjunctiva, on the basis of a dermatoscopic examination. Patients and Methods: A total of 160 conjunctival pigmented lesions were studied (40 melanomas and 120 non-melanoma conjunctival infiltrations). The clinical characteristics of the tumours were assessed with the use of dermatoscopic characteristics as described by Kittler, and with taking into consideration the typical characteristics of conjunctival lesions. Results: On the basis of modified dermatoscopic criteria, an algorithm was generated consisting of an assessment of the presence of 9 suspicious characteristics, e.g. more than two colours, colour asymmetry, pattern asymmetry, vascular polymorphism, presence of short vessels, linear vascular pattern, the presence of a peripheral structureless area, the presence of a grey structureless area and black dots in any part of the lesion. The presence of any of these characteristics scores 1 point. If a melanocytic lesion scores $\geq 3$ points, the probability of diagnosing melanoma is on the level of $p>0.001$. Conclusion: The use of the proposed algorithm, based on modified dermatoscopic characteristics, may be a valuable tool for the diagnosis of conjunctival melanoma.
\end{abstract}

Conjunctival melanoma (CMM) is a rare tumour: it is approximately 500 times less frequent than skin melanoma (1). It constitutes $1.6 \%$ of all non-cutaneous melanomas and $1-5 \%$

This article is freely accessible online.

Correspondence to: Anna Markiewicz, MD, Ph.D., Department of Ophthalmology and Ocular Oncology, Faculty of Medicine, Jagiellonian University Medical College, Kopernika 38, 31-501 Krakow, Poland. Tel: +48 124247540, Fax: +48 124247563, e-mail: anna.markiewicz@uj.edu.pl, annamarkiewicz@interia.pl

Key Words: Conjunctiva, melanoma, naevus, PAM, dermatoscopy, diagnostic algorithm. of all ocular melanomas $(2,3)$. The incidence of CMM has significantly increased in the USA and Europe over the recent years. It is estimated that the increase amongst white males is $295 \%$ within 27 years (4). Only rare cases have been reported in children $(5,6)$. The mortality rate of patients with CMM is $8 \%$ within 5 years, $13 \%$ within 8 years, and $30 \%$ within 10 years $(1,7-9)$. The prognosis of CMM is slightly better than that of melanoma of other mucosal membranes, where the 5year survival rate is $26 \%$ (10). This fact is related to the presence of CMM on a visible part of the body, which allows for early diagnosis. Conjunctival melanoma can spread through the lymphatic system and bloodstream, or it can undergo direct extension to the globe and orbit $(11,12)$. Patients with initial lymph node metastases have better prognosis than those initially presenting with systemic metastases (1). Conjunctival melanoma also has a high recurrence tendency (19-45\% within 5 years, $26-59 \%$ within 10 years and $65 \%$ within 15 years of follow-up) $(2,4,7,12-15)$. The median interval of recurrence varies between 11 and 17 months $(4,12-15)$. A poorer prognosis (higher rates of recurrence and metastases), apart from a non-bulbar tumour location, the presence of mitotic figures, epithelioid cells, lymphatic or vascular invasion, and tumour lymphangiogenesis in the histopathological examination, are typical of tumours with a thickness greater than $0.5 \mathrm{~mm}$ and with ulceration $(1,4,8,9,14)$. Therefore, it is essential to recognise CMM precursors at an early stage. A golden standard in the treatment of CMM is wide local excision (according to some authors in combination with cryotherapy to the margins and alcohol corneal epitheliectomy). Alternative or adjuvant treatments include enucleation, exenteration, topical chemotherapy (mitomycin-C, interferon-alfa2b or 5-fluorouracil), plaque brachytherapy ( $\mathrm{Ru}-$ 106 or Sr-90), proton beam radiotherapy, and external beam radiotherapy (EBRT) (2, 4, 9, 16-18). CMM must be differentiated from other melanocytic conjunctival infiltrations, such as naevi, and primary acquired melanosis (PAM), which may be CMM precursors. The correct differential diagnosis between melanoma and other melanocytic conjunctival lesions is of key significance for avoiding delays in diagnosing 
Table I. Demographic data depending on histopathological diagnosis.

\begin{tabular}{lccc}
\hline & & Histopathological diagnosis & \\
\cline { 2 - 4 } Demographic data & $\begin{array}{c}\text { Melanoma } \\
\text { (malignant tumour) } \\
(\mathrm{n}=40)\end{array}$ & $\begin{array}{c}\text { Nevus or PAM without atypia } \\
\text { (benign lesion) } \\
(\mathrm{n}=80)\end{array}$ & $\begin{array}{c}\text { PAM with atypia } \\
\text { (premalignant lesion) } \\
(\mathrm{n}=40)\end{array}$ \\
\hline $\begin{array}{l}\text { Age } \\
\text { Children }\end{array}$ & $62.7 \pm 17.7$ & $39.8 \pm 20.4$ & $59.9 \pm 17.1$ \\
Gender & $0(0.0)$ & $12(15.0)$ & $0(0.0)$ \\
Male & $18(45.0)$ & $49(61.2)$ & $16(40.0)$ \\
Female & $22(55.0)$ & $31(38.8)$ & $24(60.0)$ \\
\hline
\end{tabular}

melanoma, as this worsens the prognosis. Early stages of CMM, consisting especially of flat lesions, often pose diagnostic challenges, because at that time they share some features with benign mucosal lesions. As an incisional diagnostic biopsy of CMM may increase the risk of local recurrence of the tumour, it is necessary to search for diagnostic algorithms which would facilitate quicker diagnostics, particularly of small cancer lesions $(2,14)$. The objective of our paper was to generate a diagnostic algorithm, which would differentiate between melanomas and other melanocytic conjunctival infiltrations. This would be mainly based on the criteria applied in dermatoscopy, but also taking into consideration the characteristics that are only typical of conjunctival lesions $(19,20)$.

\section{Patients and Methods}

Patients and settings. The study was performed on consecutive nonselected pigmented melanocytic conjunctival lesions, qualified for surgical excision. It comprised 160 melanocytic conjunctival lesions in 158 patients (median age 50.5 years; age range $=5-87$ years). Among the examined tumours, a histopathological assessment confirmed 40 malignant tumours (conjunctival CMM) as well as 120 non-melanoma melanocytic conjunctival lesions (40 cases of each category: naevus, PAM without atypia and PAM with atypia). There were 83 tumours in men and 77 in women. Patients were diagnosed and treated in the Department of Ocular Oncology and Ophthalmology between 2015 and 2018. The detailed characteristics of the examined group depending on individual histopathological categories are presented in Table I.

Examined lesion diagnosis. Each conjunctival lesion, which was qualified for excision, was re-assessed by 3 independent ophthalmologists, specialising in ocular oncology, according to the previously described (19) protocol of ophthalmological examination developed for the purposes of this work. The tumours were described on the basis of eye biomicroscopy, without preliminary diagnosis. The description employed the algorithm used in dermatoscopy as described by Kittler (20). Then, a colour schematic drawing of each tumour was made with a description and a colour photo with 8,12 , and 20 -fold magnification. The images of the melanocytic lesions were obtained using a slit lamp with an SLimaging system, by a fully integrated digital camera SL Cam 5.0 (The Carl Zeiss Meditec Group, Jena, Germany). The results of the assessment were close to the contactless dermatoscopy as a diffusor filter with 8, 12, and 20-fold magnification was used. All excised lesions were examined by a pathologist with expertise in melanocytic lesions.

Clinical data. The demographic data of the patients were recorded. Then, the clinical picture of the conjunctival infiltrations was evaluated, taking into consideration the degree of their elevation (differentiating between flat, elevated, partly elevated, and flat lessions). The type and number of colours (different from the surrounding conjunctiva) of each of the infiltrations were recorded. The presence of colours was also recorded as brown (differentiating into light and dark brown), black, white, pink, grey or blue. Each lesion was scored using dermatoscopic patterns, as described by Kittler: dots, globules, pseudopods, circles, lines (the pattern is described when its core element involves a significant part of the melanocytic lesion). A structureless pattern was defined as the lack of any of the above described elements on the predominant area of the lesion (20). Then, the presence of chaos was assessed (the asymmetry of pattern or colour) in the case of all infiltrations, characterized by more than one pattern and/or colour. In addition, the vascular pattern within the infiltration was described, taking into consideration only the vessels with a different pattern from the adjacent conjunctiva and the vascular patterns described by Kittler: dots, globules, lines. The presence of short and long vessels was recorded (and short vessels were defined as those whose length is only slightly larger than their width). The presence of more than one dominating type of vessel was described as a polymorphous vessel. It was then verified whether there was any evidence of malignancy (clues of malignancy) in the melanocyte lesion as described by Kittler, such as a grey structureless area, an eccentric structureless zone (any colour except the conjunctiva), polymorphous vessels, and segmental pseudopods (20). Also, the characteristics added in our study were described, comprising cysts, peripheral feeder vessels, and black dots on the entire surface of the lesion (not only located peripherally) (19).

Statistical analysis. Continuous data are presented as a mean value and standard deviation (SD). Categorical data are presented as a percentage. Normal distribution was verified with the KolmogorovSmirnov test. Continuous data from two groups were compared with 
Table II. The comparison of the results of dermatoscopic assessment for the group with melanoma and non-melanoma melanocytic conjunctival lesions with consideration of the specificity and sensitivity of a given property.

\begin{tabular}{|c|c|c|c|c|c|}
\hline & $\begin{array}{l}\text { Non-melanoma melanocytic } \\
\text { conjunctival lesions } \\
(n=120)(\text { controls })\end{array}$ & $\begin{array}{l}\text { Conjunctival melanoma } \\
\quad(n=40) \text { (cases) }\end{array}$ & $p$-Value & $\begin{array}{l}\text { Sensitivity } \\
(\%)\end{array}$ & $\begin{array}{l}\text { Specificity } \\
(\%)\end{array}$ \\
\hline Elevated lesion & $23(19.2)$ & $15(37.5)$ & 0.018 & 37.5 & 80.8 \\
\hline Partly elevated lesion & $3(2.5)$ & $14(35.0)$ & $<0.001$ & 35.0 & 97.5 \\
\hline \multicolumn{6}{|l|}{ Pattern } \\
\hline \multicolumn{6}{|l|}{ Number of patterns } \\
\hline 1 & $31(25.8)$ & $5(12.5)$ & & & \\
\hline$\geq 2$ & $89(74.2)$ & $35(87.5)$ & & & \\
\hline Pattern asymmetry & $14(11.7)$ & $30(75.0)$ & $<0.001$ & 75.0 & 88.3 \\
\hline \multicolumn{6}{|l|}{ Type of pattern } \\
\hline Lines & $52(43.3)$ & $15(37.5)$ & 0.517 & 37.5 & 56.7 \\
\hline Dots & $104(86.7)$ & $31(77.5)$ & 0.167 & 77.5 & 13.3 \\
\hline Papules & $5(4.2)$ & $5(12.5)$ & 0.123 & 12.5 & 95.8 \\
\hline Circles & $6(5.0)$ & $1(2.5)$ & 0.681 & 2.5 & 95.0 \\
\hline Pseudopods & $1(0.8)$ & $7(17.5)$ & $<0.001$ & 17.5 & 99.2 \\
\hline Structureless & $53(44.2)$ & $39(97.5)$ & $<0.001$ & 97.5 & 55.8 \\
\hline \multicolumn{6}{|l|}{ Colour } \\
\hline \multicolumn{6}{|l|}{ Number of colours } \\
\hline 1 & $60(50.0)$ & $2(5.0)$ & & & \\
\hline 2 & $44(36.7)$ & $11(27.5)$ & & & \\
\hline 3 & $15(12.5)$ & $17(42.5)$ & & & \\
\hline 4 & $1(0.8)$ & $9(22.5)$ & & & \\
\hline 5 & $0(0.0)$ & $0(0.0)$ & & & \\
\hline 6 & $0(0.0)$ & $1(2.5)$ & & & \\
\hline Colour asymmetry & $14(11.7)$ & $31(77.5)$ & $<0.001$ & 77.5 & 88.3 \\
\hline Light brown & $81(67.5)$ & $25(62.5)$ & 0.562 & 62.5 & 32.5 \\
\hline Dark brown & $65(54.2)$ & $27(67.5)$ & 0.140 & 67.5 & 45.8 \\
\hline Black & $29(24.2)$ & $23(57.5)$ & $<0.001$ & 57.5 & 75.8 \\
\hline White & $0(0.0)$ & $6(15.0)$ & $<0.001$ & 15.0 & 100.0 \\
\hline Grey/blue & $11(9.2)$ & $19(47.5)$ & $<0.001$ & 47.5 & 90.8 \\
\hline Pink & $11(9.2)$ & $16(40.0)$ & $<0.001$ & 40.0 & 90.8 \\
\hline \multicolumn{6}{|l|}{ Blood vessels } \\
\hline \multicolumn{6}{|l|}{ Symmetry of vascular pattern } \\
\hline Polymorphic & $7(5.8)$ & $20(50.0)$ & & & \\
\hline Monomorphic & $21(17.5)$ & $6(15.0)$ & & & \\
\hline None & $92(76.7)$ & $14(35.0)$ & & & \\
\hline \multicolumn{6}{|l|}{ Type of vascular pattern } \\
\hline Dots & $6(5.0)$ & $16(40.0)$ & $<0.001$ & 40.0 & 95.0 \\
\hline Papules & $2(1.7)$ & $4(10.0)$ & 0.035 & 10.0 & 98.3 \\
\hline Lines & $26(21.7)$ & $25(62.5)$ & $<0.001$ & 62.5 & 78.3 \\
\hline The presence of short vessels & $15(12.5)$ & $24(60.0)$ & $<0.001$ & 60.0 & 87.5 \\
\hline The presence of long vessels & $19(15.8)$ & $18(45.0)$ & $<0.001$ & 45.0 & 84.2 \\
\hline \multicolumn{6}{|c|}{ Clues to malignancy and other special properties } \\
\hline Peripheral structureless area & $4(3.3)$ & $11(27.5)$ & $<0.001$ & 27.5 & 96.7 \\
\hline Grey structureless area & $11(9.2)$ & $20(50.0)$ & $<0.001$ & 50.0 & 90.8 \\
\hline Black dots & $29(24.2)$ & $22(55.0)$ & $<0.001$ & 55.0 & 75.8 \\
\hline Segmental pseudopods & $1(0.8)$ & $8(20.0)$ & $<0.001$ & 20.0 & 99.2 \\
\hline Peripheral feeder vessels & $4(3.3)$ & $16(40.0)$ & $<0.001$ & 40.0 & 96.7 \\
\hline Cysts & $14(11.7)$ & $0(0.0)$ & 0.022 & 0.0 & 88.3 \\
\hline
\end{tabular}

Bold values indicate statistical significance. 
the Student's $t$-test or the Mann-Whitney $U$-test, depending on the distribution. More than two groups were compared with the univariate analysis of variance (ANOVA) followed by the Least Significant Difference (LSD) test or with the Kruskall-Wallis test depending on the distribution. Categorical data were compared with the Chi-square test and Fisher's exact test. The receiver operating characteristic (ROC) curve was performed to assess the area under the curve (AUC), as well as the sensitivity and specificity of our melanoma scoring. A $p$-value less than 0.05 was considered statistically significant. Data were analysed with the use of SPSS software v.21 (IBM, Armonk, NY, USA).

The authors declare that this investigation was carried out according to the rules of the Declaration of Helsinki of 1975 (revised in 2013).

\section{Results}

Table II contains the results of the study on the basis of the guidelines used in dermatoscopy. The comparative analyses of the results of the examinations carried out on the group of patients with conjunctival melanoma versus the control group (other melanocytic conjunctival infiltrations) revealed many significant results (Table II). In both groups, there were completely elevated infiltrations, yet CMM was more often found only in partly elevated tumours $(p<0.001)$. The studied groups did not differ from each other with regards to the number of patterns observed; in both groups the dominating pattern comprised dots and lines. In melanoma, however, pattern asymmetry was more frequent $(p<0.001)$; similarly to the structureless pattern and pseudopods $(p<0.001)$. In both study groups, the dominating colour was light and dark brown, CMM however, had a larger average number of colours and colour asymmetry $(p<0.001)$ and also more frequently contained the colour black, white, grey, or blue and pink $(p<0.001)$. In the CMM group, evidence of malignancy comprising a peripheral structureless area, a grey structureless area, black dots (not only located peripherally but also on the entire area of the tumour), as well as segmentally located pseudopods and polymorphous vessels $(p<0.001)$ were statistically more frequent. Cysts were more typical for nonmelanoma conjunctival infiltrations; their presence was found in none of the cases of CMM, yet this was not statistically significant. The control group of non-melanoma tumours did not have visible vascularisation, and if visible vessels existed, they rather had a homogenous pattern (monomorphic) $(p<0.001)$. In the case of CMM, characteristics such as visible vascularisation with the presence of both short and long vessels as well as polymorphous and peripheral feeder vessels were seen more frequently $(p<0.001)$.

With the use of statistical analysis tools, we generated a new algorithm for the diagnosis of melanoma in the case of melanocytic conjunctival lesions (Table III). Each of the following characteristics score 1 point: the presence of more than two colours, colour asymmetry, pattern asymmetry, vascular polymorphism, the presence of short vessels, a linear
Table III. The list of the properties evaluated in algorithm for pigmented conjunctival lesions. A lesion is considered as suspicious and should be removed if the score is $\geq 3$.

\begin{tabular}{lc}
\hline Characteristics of algorithm & Points \\
\hline More than two colours & 1 \\
Colour asymmetry & 1 \\
Pattern asymmetry & 1 \\
Polymorphous vessels & 1 \\
Vessels lines pattern & 1 \\
Short vessels & 1 \\
Eccentric structureless zone & 1 \\
Grey structureless pattern & 1 \\
Black dots & 1 \\
\hline
\end{tabular}

vascular pattern, the presence of a peripheral structureless area (in any colour different from the colour of the surrounding conjunctiva), the presence of grey structureless area and black dots (in any part of the lesion) (Figures 1 and 2).

The ROC for melanoma is illustrated in Figure 3. The diagnostic strength of AUC was $0.918(p<0.001)$. For the cut-off point of 3.0, the sensitivity of the test was $90 \%$, and specificity $83 \%$.

The scoring of the presence of specific properties indicative of malignancy in various types of melanocytic conjunctival lesions is presented in Table IV.

On the basis of the scoring, only the differentiation between the diagnosis of PAM with atypia and melanocytic naevus was uncertain $(p=0.681)$; in all other cases (the differentiation between PAM without atypia and PAM with atypia or naevus or melanoma as well as melanoma and PAM with atypia or naevus) it was very certain $(p<0.001)$.

\section{Discussion}

In dermatology, there are many dermatoscopic algorithms that facilitate the diagnosis of skin melanoma. It is known, that a dermatoscopic picture depends on the tumour location on the skin. For mucosal membranes (other than conjunctiva), the literature describes the presence of many dermatoscopic characteristics suggesting the presence of melanoma, such as a multicomponent pattern, an irregular pattern, the presence of white, grey or blue colour, particularly in connection with a structureless area, atypical network, polychrome, irregular dots or grey granulation, atypical or irregular vessels or bluewhite vail $(2,21-25)$. These data, however, cannot be used for the diagnosis of conjunctival melanoma, based on the differences in structure compared to other mucosal membranes. To the best of our knowledge, so far there has not been any simple algorithm for the diagnosis of the early form of conjunctival melanoma. Shields et al., in their study of a large group of conjunctival tumours, observed that the 


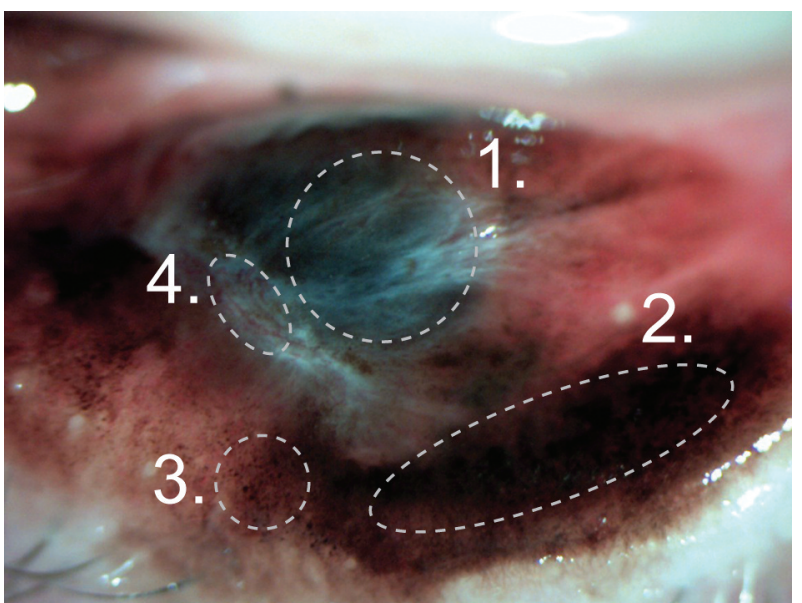

Figure 1. Conjunctival melanoma - with more than 2 visible colours (grey, white, pink and dark brown), partly flat and partly elevated infiltration [1-grey structureless; 2-peripheral structureless area (brown colour); 3-black dots; 4-vessels lines].

greatest relative risks for melanoma (vs. PAM) were older age, tarsal location, lack of pigmentation, greater than $1 \mathrm{~mm}$ thickness, feeder vessels, cysts, intrinsic vessels, and haemorrhaging (26). However, many of these characteristics are related to advanced cancer. Nevertheless, little is known about the possible role of dermatoscopic algorithms in the diagnosis of conjunctival melanoma. In the first study by Cinotti et al. concerning the dermatoscopic features of a series of conjunctival lesions, the authors could not find any dermatoscopic criteria allowing for the differential diagnosis of melanoma, except for the higher presence of a grey colour in CMM than in naevi (27). Tosi et al. described 39 conjunctival pigmented tumours that were imaged with a noncontact camera that provided digital images at $16 x$ magnification, and evaluated many dermatoscopic parameters using a dedicated software (digital surface dermoscopy). CMM was found to be larger, darker, and with more internal contrast and blue-grey colour than benign lesions (24).

However, no large-scale study has been performed to define the dermatoscopy criteria for conjunctival melanoma. We evaluated whether the algorithms commonly used for distinguishing melanoma from benign lesions of the skin are also applicable to lesions on the conjunctiva. We managed to confirm earlier reports about the more frequent presence of grey or blue colour in the case of conjunctival melanoma. Additionally, we showed that other properties of the pigmentation of a melanocytic tumour such as the presence of a black, white, and pink colour, and a larger average number of colours and colour asymmetry point to its malignant character. We also described reliable dermatoscopic patterns typical of CMM, such as a structureless pattern and pattern

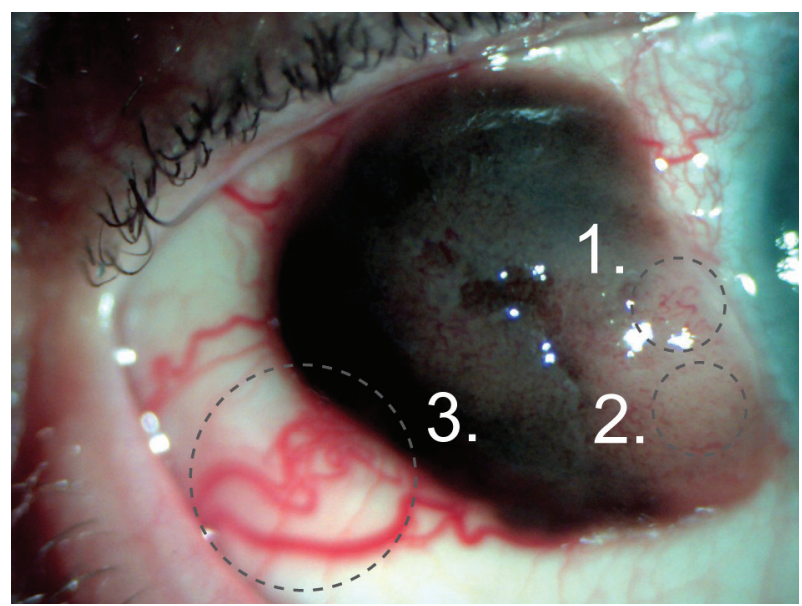

Figure 2. Conjunctival melanoma - visible polymorphous vessels (1linear long vessels; 2-short vessels; 3-feeder vessels).

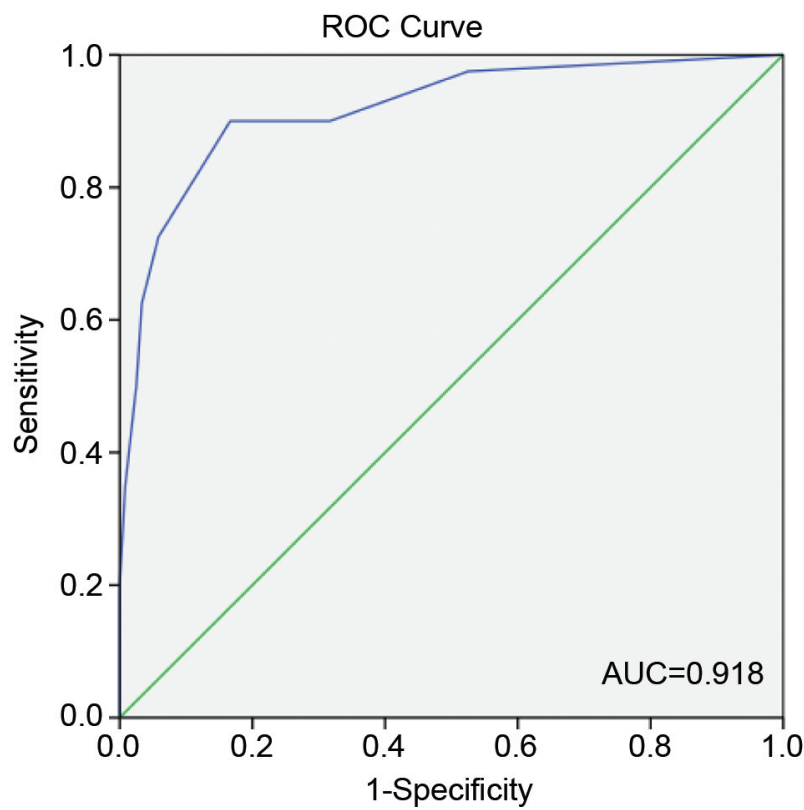

Figure 3. Algorithm presenting receiver operating characteristic (ROC) curve for the diagnosis of melanoma. The ROC curve was performed to assess the area under the curve (AUC), sensitivity, and specificity of our melanoma scoring. A p-value $<0.05$ was considered statistically significant.

asymmetry. Furthermore, we documented evidence of malignancy comprising a peripheral structureless area, a grey structureless area, black dots (not only located peripherally but also on any part of the tumour), and segmentally located pseudopods, which are all typical of CMM. In addition, we 
Table IV. Quantitative results for the presence of specific properties indicative of malignancy (scoring) $w$ in various types of conjunctival melanocytic lesions.

\begin{tabular}{lcccc}
\hline & $\begin{array}{c}1 \\
\text { PAM without atypia } \\
(\mathrm{n}=40)\end{array}$ & $\begin{array}{c}2 \\
\text { PAM with atypia } \\
(\mathrm{n}=40)\end{array}$ & $\begin{array}{c}3 \\
\text { Naevus } \\
(\mathrm{n}=40)\end{array}$ & $\begin{array}{c}4 \\
\text { Melanoma } \\
(\mathrm{n}=40)\end{array}$ \\
\hline Scoring & $0.1 \pm 0.4$ & $1.7 \pm 1.5$ & $1.6 \pm 1.6$ & $5.3 \pm 2.4$ \\
\hline
\end{tabular}

observed the more frequent presence of visible vascularisation of the lesion in melanoma as compared with benign melanocytic infiltrations of the conjunctiva. In CMM, both short and long vessels as well as polymorphous and peripheral feeder vessels were presented.

We also created a simple and reliable diagnostic algorithm for detecting CMM in its early stage. The algorithm assesses the presence of 9 suspicious characteristics, which are referred to in Table III. Each characteristic scores 1 point. If the clinical assessment of a given melanocytic lesion scores $\geq 3$ points, the probability of diagnosing melanoma is on the level of $p>0.001$.

The use of the proposed algorithm that is based on dermatoscopic characteristics appears to be a valuable tool for the diagnosis of conjunctival melanoma. However, further larger collaborative studies are needed to better validate our conjunctival melanoma diagnostic algorithm.

\section{Conflicts of Interest}

The Authors declare no conflicts of interest in relation to this study.

\section{Authors' Contributions}

M.D-K.: Conceptualization, methodology, investigation, data curation, writing - original draft, visualization, formal analysis; B.R-D.: conceptualization, methodology, investigation, data curation, writing review \& editing; J.K.: methodology, writing - review \& editing, investigation, visualization; I.K-B.: methodology, writing -review \& editing, investigation; W.P-M.: writing- review \& editing, data curation, investigation; A.M.: conceptualization, methodology, investigation, supervision; writing - original draft; writing - review \& editing; project administration, formal analysis, data curation. All Authors have reviewed the article and approved the submitted version.

\section{References}

1 Brownstein S: Malignant melanoma of the conjunctiva. Cancer Control 11(5): 310-316, 2004. PMID: 15377990. DOI: $10.1177 / 107327480401100505$

2 Shields CL: Conjunctival melanoma. Br J Ophthalmol 86(2): 127, 2002. PMID: 11815331. DOI: 10.1136/bjo.86.2.127

3 Volpini BMF, Maia M, Agi J, Vital J Filho and Lellis RF: Synchronous conjunctival melanoma and lentigo maligna melanoma. An Bras Dermatol 92(4): 565-567, 2017. PMID: 28954115. DOI: 10.1590/abd1806-4841.20176015
4 Oellers P and Karp C: Management of pigmented conjunctival lesions. The Ocular Surface 10(4): 251-263, 2018. DOI: 10.1016/j.jtos.2012.08.002

5 Romanowska-Dixon B, Jager MJ and Coupland SE: Onkologia okulistyczna. $1^{\text {st }}$ Edition. Warsaw, PZWL Wydawnictow Lekarskie, 2019.

6 Rakusiewicz K, Kanigowska K, Grajkowska W and Hautz W: Surgical treatment of conjunctival nevi in pediatric patients. Klinika Oczna 2019(1): 29-33, 2019. DOI: 10.5114/ko.2019.85213

7 Shields CL and Shields JA: Tumors of the conjunctiva and cornea. Indian J Ophthalmol 67(12): 1930-1948, 2019. PMID: 31755426. DOI: 10.4103/ijo.IJO_2040_19

8 Novais GA, Fernandes BF, Belfort RN, Castiglione E, Cheema DP and Burnier MN Jr: Incidence of melanocytic lesions of the conjunctiva in a review of 10675 ophthalmic specimens. Int J Surg Pathol 18(1): 60-63, 2010. PMID: 18611943. DOI: $10.1177 / 1066896908319775$

9 Furdova A, Pesko K, Strmen P and Kobzova M: Conjunctival nevus and melanoma. Bratisl Lek Listy 108(7): 287-291, 2007. PMID: 17972544.

$10 \mathrm{Li} \mathrm{K}$ and $\mathrm{Xin} \mathrm{L}$ : Palpebral conjunctiva melanoma with dermoscopic and clinicopathological characteristics. J Am Acad Dermatol 71(2): e35-e37, 2014. PMID: 25037804. DOI: 10.1016/j.jaad.2013.11.011

11 Albreiki DH, Gilberg SM and Farmer JP: Conjunctival malignant melanoma: A rare variant and review of important diagnostic and therapeutic considerations. Saudi J Ophthalmol 26(2): 151-156, 2012. PMID: 23960986. DOI: 10.1016/j.sjopt.2012.02.006

12 Zhou C, Wang Y, Jia R and Fan X: Conjunctival melanoma in Chinese patients: Local recurrence, metastasis, mortality, and comparisons with Caucasian patients. Invest Ophthalmol Vis Sci 58(12): 5452-5459, 2017. PMID: 29059312. DOI: 10.1167/iovs.1722376

13 Shields CL, Markowitz JS, Belinsky I, Schwartzstein H, George NS, Lally SE, Mashayekhi A and Shields JA: Conjunctival melanoma: outcomes based on tumor origin in 382 consecutive cases. Ophthalmology 118(2): 389-95.e1-2, 2011. PMID: 20723990. DOI: 10.1016/j.ophtha.2010.06.021

14 Jain P, Finger PT, Fili M, Damato B, Coupland SE, Heimann H, Kenawy N, J Brouwer N, Marinkovic M, Van Duinen SG, Caujolle JP, Maschi C, Seregard S, Pelayes D, Folgar M, Yousef YA, Krema H, Gallie B, Calle-Vasquez A and American Joint Committee on Cancer Ophthalmic Oncology Task Force: Conjunctival melanoma treatment outcomes in 288 patients: a multicentre international data-sharing study. Br J Ophthalmol, 2020. PMID: 32892167. DOI: 10.1136/bjophthalmol-2020-316293

15 Esmaeli B, Wang X, Youssef A and Gershenwald JE: Patterns of regional and distant metastasis in patients with conjunctival melanoma: experience at a cancer center over four decades. 
Ophthalmology 108(11): 2101-2105, 2001. PMID: 11713086. DOI: $10.1016 / \mathrm{s} 0161-6420(01) 00782-5$

16 Shields CL and Shields JA: Tumors of the conjunctiva and cornea. Indian J Ophthalmol 67(12): 1930-1948, 2019. PMID: 31755426. DOI: 10.4103/ijo.IJO_2040_19

17 Brownstein S: Malignant melanoma of the conjunctiva. Cancer Control 11(5): 310-316, 2004. PMID: 15377990. DOI: $10.1177 / 107327480401100505$

18 Albreiki DH, Gilberg SM and Farmer JP: Conjunctival malignant melanoma: A rare variant and review of important diagnostic and therapeutic considerations. Saudi J Ophthalmol 26(2): 151-156, 2012. PMID: 23960986. DOI: 10.1016/j.sjopt.2012.02.006

19 DĘbicka-Kumela M, Romanowska-Dixon B, Karska-Basta I, Kowal $\mathrm{J}$ and Markiewicz A: The evaluation of the malignant characteristics of conjunctival lesions based on the dermatoscopic algorithm. Anticancer Res 41(2): 895-903, 2021. PMID: 33517295. DOI: 10.21873/anticanres.14842

20 Kittler H, Rosendahl C, Cameron A and Tschandl P: Deramatoscopy: An algorithmic method based on pattern analysis. Gdańsk, Via Medica, pp. 40-165, 206, 2012.

21 Blum A, Simionescu O, Argenziano G, Braun R, Cabo H, Eichhorn A, Kirchesch H, Malvehy J, Marghoob AA, Puig S, Ozdemir F, Stolz W, Tromme I, Weigert U, Wolf IH, Zalaudek I and Kittler H: Dermoscopy of pigmented lesions of the mucosa and the mucocutaneous junction: results of a multicenter study by the International Dermoscopy Society (IDS). Arch Dermatol 147(10): 1181-1187, 2011. PMID: 21680757. DOI: 10.1001/ archdermatol.2011.155

22 Ronger-Savle S, Julien V, Duru G, Raudrant D, Dalle S and Thomas L: Features of pigmented vulval lesions on dermoscopy. Br J Dermatol 164(1): 54-61, 2011. PMID: 20846309. DOI: 10.1111/j.1365-2133.2010.10043.x
23 Rogers T, Pulitzer M, Marino ML, Marghoob AA, Zivanovic O and Marchetti MA: Early diagnosis of genital mucosal melanoma: how good are our dermoscopic criteria? Dermatol Pract Concept 6(4): 43-46, 2016. PMID: 27867747. DOI: $10.5826 / \mathrm{dpc} .0604 \mathrm{a} 10$

24 Tosi GM, Rubegni P, Schuerfeld K, Toti P, Cevenini G, Dell'Eva G, Andreassi L, Caporossi A and Burroni M: Digital surface microscopy analysis of conjunctival pigmented lesions: a preliminary study. Melanoma Res 14(5): 375-380, 2004. PMID: 15457093. DOI: 10.1097/00008390-200410000-00007

25 Lin J, Koga H, Takata M and Saida T: Dermoscopy of pigmented lesions on mucocutaneous junction and mucous membrane. Br J Dermatol 161(6): 1255-1261, 2009. PMID: 19673880. DOI: 10.1111/j.1365-2133.2009.09251.x

26 Shields CL, Alset AE, Boal NS, Casey MG, Knapp AN, Sugarman JA, Schoen MA, Gordon PS, Douglass AM, Sioufi K, Say EA and Shields JA: Conjunctival tumors in 5002 cases. Comparative analysis of benign versus malignant counterparts. The 2016 James D. Allen lecture. Am J Ophthalmol 173: 106133, 2017. PMID: 27725148. DOI: 10.1016/j.ajo.2016.09.034

27 Cinotti E, La Rocca A, Labeille B, Grivet D, Tognetti L, Lambert V, Kaspi M, Nami N, Fimiani M, Perrot JL and Rubegni P: Dermoscopy for the diagnosis of conjunctival lesions. Dermatol Clin 36(4): 439-449, 2018. PMID: 30201153. DOI: $10.1016 /$ j.det.2018.05.011
Received April 28, 2021

Revised May 11, 2021

Accepted May 13, 2021 